\title{
Effect of adaptive cluster sampling design on accuracy of sediment rating curve estimation.
}

\begin{abstract}
Adaptive cluster sampling represents a design whereby more samples during high storm hydrographs can be obtained in the field compared to the commonly used calendar-based. To compare the effect of these sampling designs on accuracy of sediment rating curve estimation, we performed a study for Gorgan-Rood River, Iran by synthesizing sample sets from daily records. The load estimates obtained by conventional, two bias-corrected, and logged mean load within discharge classes (LMLWDCs) rating curves were statistically evaluated. All rating curves derived from calendar-based sample sets-except those with a nonparametric correction factor-underestimated the average load from 25 to $76 \%$. Rating curves derived from adaptive sample sets, however, increased the sediment load to as much as $30 \%$. Among this group of rating curves, LMLWDC produced the most accurate results with only $3 \%$ overestimation and a coefficient of variation in the order of $14 \%$ when the sampling frequency was semiweekly. The more accurate estimates from adaptive sample sets are likely due to the inclusion of more samples from high load periods.
\end{abstract}

Keyword: Adaptive cluster sampling; Sediment load estimation; Sediment rating curve; Gorgan-Rood River; Iran. 